\title{
Application of Reproducing Kernel Hilbert Space Method for Solving a Class of Nonlinear Integral Equations
}

\author{
Sedigheh Farzaneh Javan, ${ }^{1}$ Saeid Abbasbandy, ${ }^{1}$ and M. Ali Fariborzi Araghi ${ }^{2}$ \\ ${ }^{1}$ Department of Mathematics, Science and Research Branch, Islamic Azad University, Tehran 14515, Iran \\ ${ }^{2}$ Department of Mathematics, Central Tehran Branch, Islamic Azad University, Tehran, Iran \\ Correspondence should be addressed to M. Ali Fariborzi Araghi; fariborzi.araghi@gmail.com
}

Received 11 November 2016; Revised 7 February 2017; Accepted 8 February 2017; Published 16 March 2017

Academic Editor: Haipeng Peng

Copyright (c) 2017 Sedigheh Farzaneh Javan et al. This is an open access article distributed under the Creative Commons Attribution License, which permits unrestricted use, distribution, and reproduction in any medium, provided the original work is properly cited.

\begin{abstract}
A new approach based on the Reproducing Kernel Hilbert Space Method is proposed to approximate the solution of the secondkind nonlinear integral equations. In this case, the Gram-Schmidt process is substituted by another process so that a satisfactory result is obtained. In this method, the solution is expressed in the form of a series. Furthermore, the convergence of the proposed technique is proved. In order to illustrate the effectiveness and efficiency of the method, four sample integral equations arising in electromagnetics are solved via the given algorithm.
\end{abstract}

\section{Introduction}

Electromagnetics is the phenomenon associated with electric and magnetic fields and their interactions which is generally one of the most important sciences. Exterior calculus is given in $[1,2]$ inside some textbooks. A way to teach electromagnetics can be approached via the use of differential forms which is given in [3]. According to electromagnetic field problems from many years ago, some solutions via linear and nonlinear integral equations (NIE) have been given which can be useful in the field. In those methods like block-pulse functions (BPFs), Galerkin, and collocation, the most important ways are basic functions and appropriate projection. Based on the Reproducing Kernel Hilbert Space method, an approach has been found to solve some electromagnetic issues.

Nonlinear integral equations are encountered in different fields of science and numerous applications as elasticity, plasticity, heat and mass transfer, oscillation theory, fluid dynamics, filtration theory, electrostatics, electrodynamics, biomechanics, game theory, control, queuing theory, electrical engineering, economics, and medicine, among others. There are different types of NIE usually which cannot be worked out explicitly, so it should be approached approximately.

Therefore, many researchers studied and focused on different numerical techniques which can work out these integral equations. For instance, in $[4,5]$, the authors presented the homotopy analysis method to solve the second kind of nonlinear Fredholm and Volterra integral equations. The linear multistep techniques were applied in [6], to obtain the numerical solution of a singular nonlinear Volterra integral equation. In [7], an asymptotic technique to approach numerically the nonlinear Abel-Volterra integral equation was applied.

Reproducing Kernel Hilbert Space (RKHS) was introduced by Minggen et al. [8,9], and it was developed in different areas, including approximation theory, statistics, machine learning theory, group representation theory, and various areas of complex analysis. Reproducing Kernel Hilbert Space Method (RKHSM) is a kernel based approximation method which was applied for solving nonlinear boundary value problems [7-12], generalized singular nonlinear Lane-Emden type equations [13], integrodifferential equations [14-16], integrodifferential fractional equations [17], Bratus Problem [18], and so forth.

Consider the following nonlinear integral equation:

$$
u(x)+\int_{a}^{b(\text { or } x)} K(x, t) N(u(t)) d t=f(x)
$$


where $a, b$ are real constants, $u \in W_{2}^{1}[a, b]$ is an unknown function which can be determined, $f \in W_{2}^{1}[a, b]$ is a continuous function on $[a, b], K(x, t)$ is a continuous function on $[a, b] \times[a, b], N(v(x))$ is a continuous term in $W_{2}^{1}[a, b]$ as $v(x) \in W_{2}^{1}[a, b], a \leq x \leq b$, and $W_{2}^{1}[a, b]$ is Reproducing Kernel Space. Equation (1) has a continuous solution on $[a, b]$ [19]. The existence and uniqueness conditions of the solution for (1) were discussed in [19-23]. We assume that the solution of (1) is unique.

Over several decades, numerical methods in electromagnetic problems have been one of the most important subjects of extensive researches [1-4]. On the other hand, many problems in electromagnetics can be modeled by integral equations mentioned in [24-26], for example, electric field integral equation (EFIE) and magnetic field integral equation (MFIE). In recent years, several numerical methods for solving linear and nonlinear integral equations have been presented. Applicable equations of electromagnetics have been implied in the presented paper.

In previous works like [13-15], the Gram-Schmidt orthogonalization process has been considered to implement RKHSM. Since this process is unstable numerically and it may take a lot of time to run the algorithm, here, we put away this process and act with another way. Our approach combines the methods mentioned in [13-17]. More specifically, on the contrary to [13-15], without use of the orthogonalization process, the RKSHM is applied successfully to solve the nonlinear problem (1).

The structure of this paper would be described as follows. In Section 2, the basic definitions, assumptions, and preliminaries of RKHS are described. The main idea and convergence of the proposed scheme are discussed in Section 3. Section 4 contains the numerical experiments. Finally, Section 5 is dedicated to a brief conclusion.

\section{Preliminaries}

In this section, some basic definitions and important properties of Reproducing Kernel Hilbert Spaces (RKHS) are mentioned [8, 9, 27-29].

Definition 1. A Hilbert Space $H$ is an inner product space that is complete and separable with respect to the norm defined by the inner product. Completeness of the space $H$ holds provided that every Cauchy sequence of points in $H$ that has a limit that is also in $H$ and separable of $H$ admits a countable orthonormal basis of it.

Definition 2. For an abstract set $\mathscr{X}$, let $\mathscr{H}$ be a Hilbert Space of real or complex-valued functions on set $\mathscr{X}$. We say $\mathscr{H}$ is a Reproducing Kernel Hilbert Space if there exist a linear and bounded evaluation functional $R_{x}$ over $\mathscr{H}$, or, equivalently,

$$
R_{x}: f \longmapsto f(x) \quad \forall f \in H .
$$

Riesz Representation Theorem implies that for all $x$ in $\mathscr{X}$ there exists a unique function $K_{x}$ of $\mathscr{H}$ with the reproducing property,

$$
f(x)=R_{x}(f)=\left\langle f(y), R_{x}(y)\right\rangle_{\mathscr{H}} \quad \forall f \in \mathscr{H},
$$

for each $y \in \mathscr{H}$ where $\langle\cdot, \cdot\rangle_{\mathscr{H}}$ represent the inner product of the Hilbert Space $\mathscr{H}$.

Definition 3. The space $W_{2}^{m}[a, b]$ is interpreted as

$$
\begin{gathered}
W_{2}^{m}[a, b]=\left\{u(x) \mid u:[a, b] \rightarrow R, u^{(m-1)}\right. \\
\left.\in A C[a, b], u^{(m)} \in L^{2}[a, b]\right\}
\end{gathered}
$$

The inner product and the norm in $W_{2}^{m}[a, b]$ are of forms

$$
\begin{aligned}
\langle u(x), v(x)\rangle_{W_{2}^{m}}= & \sum_{i=0}^{m-1} u^{(i)}(a) v^{(i)}(a) \\
& +\int_{a}^{b} u^{(m)}(\xi) v^{(m)}(\xi) d \xi, \\
& u, v \in W_{2}^{m}[a, b], \\
\|u\|=\sqrt{\langle u(x), v(x)\rangle_{W_{2}^{m}}}, & \\
& u \in W_{2}^{m}[a, b] .
\end{aligned}
$$

Lemma 4 (see $[9,29])$. Functional space $W_{2}^{m}[a, b]$ is inner space.

Theorem 5 (see $[9,29])$. Functional space $W_{2}^{m}[a, b]$ is a Hilbert Space.

Theorem 6 (see $[9,29])$. Functional space $W_{2}^{m}[a, b]$ is Reproducing Kernel Hilbert Space.

Now, it is taken away that expression form of the Reproducing Kernel function $R_{x}(t) \in W_{2}^{m}[a, b]$.

Based on essay, it is easy to prove that $R_{x}(t)$ is the answer of the following generalized differential equation $[9,29]$ :

$$
\begin{aligned}
(-1)^{m} \frac{\partial^{2 m} R_{x}(t)}{\partial t^{2 m}} & =\delta(t-x), \\
\frac{\partial^{i} R_{y}(a)}{\partial t^{i}}-(-1)^{m-i-1} \frac{\partial^{2 m-i-1} R_{y}(a)}{\partial t^{2 m-i-1}} & =0, \\
\frac{\partial^{2 m-i-1} R_{y}(b)}{\partial t^{2 m-i-1}} & =0, \\
i & =0,1, \ldots, m-1,
\end{aligned}
$$

where $\delta$ is Dirac's delta function. While $x \neq t, R_{x}(t)$ is the answer of the following constant linear homogeneous differential equation with $2 m$ order:

$$
(-1)^{m} \frac{\partial^{2 m} R_{x}(t)}{\partial t^{2 m}}=0
$$


with the boundary conditions

$$
\begin{aligned}
\frac{\partial^{i} R-y(a)}{\partial t^{i}}-(-1)^{m-i-1} \frac{\partial^{2 m-i-1} R_{y}(a)}{\partial t^{2 m-i-1}} & =0, \\
\frac{\partial^{2 m-i-1} R_{y}(b)}{\partial t^{2 m-i-1}} & =0, \\
i & =0,1, \ldots, m-1 .
\end{aligned}
$$

Equation (7) is characteristic $\lambda^{2 m}=0$. Then the general solution of Equation (7) is

$$
R_{x}(t)= \begin{cases}\sum_{i=1}^{2 m} c_{i}(x) t^{i-1}, & t \leq x, \\ \sum_{i=1}^{2 m} d_{i}(x) t^{i-1}, & t>x,\end{cases}
$$

where coefficients $c_{i}(x)$ and $d_{i}(x), i=1,2, \ldots, 2 m$, could be calculated by solving the following linear equations:

$$
\begin{aligned}
& \frac{\partial^{i} R_{x}(x+0)}{\partial t^{i}}=\frac{\partial^{i} R_{x}(x-0)}{\partial t^{i}}, \quad i=0,1, \ldots, 2 m-2, \\
& \left(\frac{\partial^{2 m-1} R_{x}(x+0)}{\partial t^{2 m-1}}-\frac{\partial^{2 m-1} R_{x}(x-0)}{\partial t^{2 m-1}}\right)=(-1)^{m}, \\
& \frac{\partial^{i} R_{y}(a)}{\partial t^{i}}-(-1)^{m-i-1} \frac{\partial^{2 m-i-1} R_{y}(a)}{\partial t^{2 m-i-1}}=0, \\
& \frac{\partial^{2 m-i-1} R_{y}(b)}{\partial t^{2 m-i-1}}=0, \quad i=0,1, \ldots, m-1 .
\end{aligned}
$$

Subsequently, the representation of the Reproducing Kernel of $W_{2}^{1}[a, b]$ is provided by

$$
R_{x}(t)= \begin{cases}1-a+t, & t \leq x, \\ 1-a+x, & t>x .\end{cases}
$$

\section{Main Idea and Theoretical Discussion}

The uniqueness conditions for nonlinear problems exist in [21-23]. The unique solution of (1) is assumed in this paper. The solution of (1) is given in $W_{2}^{1}[a, b]$ space. We consider (1) as

$$
\llbracket u(x)=u(x),
$$

where $\llbracket u(x)=f(x)-\int_{a}^{b(\operatorname{or} x)} K(x, t) N(u(t)) d t$. It is obvious that $\mathbb{L}$ is the bounded linear operator of $W_{2}^{1}$ to $W_{2}^{1}$. Put $\varphi_{i}(x)=$ $R_{x_{i}}(x)$ and $\psi_{i}(x)=\mathbb{L}^{*} \varphi_{i}(x)$, where $\mathbb{L}^{*}$ is the adjoint operator of $\mathbb{L}$. In fact, for $u, v \in W_{2}^{1}[a, b]$, we have $\mathbb{L}(u(x)+v(x))=$ $u(x)+v(x)$ and $\|\mathbb{L} u(x)\|_{W_{2}^{1}[a, b]}=\|u(x)\|_{W_{2}^{1}[a, b]}$.

The orthonormal system of $\left\{\bar{\psi}_{i}(x)\right\}_{i=1}^{\infty}$ from the space $W_{2}^{1}[a, b]$ can be derived from Gram-Schmidt orthogonal process of $\left\{\psi_{i}(x)\right\}_{i=1}^{\infty}$ :

$$
\bar{\psi}_{i}(x)=\sum_{k=1}^{i} \beta_{i k} \psi_{k}(x), \quad \beta_{i i}>0, i=1,2, \ldots
$$

Definition 7. In a topological space $(\mathscr{X}, \tau)$, a subset $A$ of $\mathscr{X}$ is called dense in $\mathscr{X}$ if $\bar{A}=\operatorname{cl} A=\mathscr{X}$.

Theorem 8. If $\left\{x_{i}\right\}_{i=1}^{\infty}$ is dense on $[a, b]$ then $\left\{\psi_{i}(x)\right\}_{i=1}^{\infty}$ is the complete function system of the space $W_{2}^{1}[a, b]$ and $\psi_{i}(x)=$ $\left.L_{t} R_{x}(t)\right|_{t=x_{i}}$, where the subscript $t$ in the operator $\mathbb{L}$ indicates that the operator $\mathbb{L}$ applies to the function of $t$.

Proof. We have

$$
\begin{aligned}
\psi_{i}(x) & =\left\langle\left(\mathbb{L}^{*} \varphi_{i}\right)(t), R_{x}(t)\right\rangle_{W_{2}^{1}[a, b]} \\
& =\left\langle\varphi_{i}(t), L_{t} R_{x}(t)\right\rangle_{W_{2}^{1}[a, b]}=\left.L_{t} R_{x}(t)\right|_{t=x_{i}} .
\end{aligned}
$$

Clearly $\psi_{i}(x) \in W_{2}^{1}[a, b]$. For each fixed $u(x) \in W_{2}^{1}[a, b]$, let $\left\langle u(x), \psi_{i}(x)\right\rangle_{W_{2}^{1}[a, b]}=0, i=1,2, \ldots$, which means

$$
\begin{aligned}
& \left\langle u(x),\left(\mathbb{L}^{*} \varphi_{i}\right)(x)\right\rangle_{W_{2}^{1}[a, b]}=\left\langle\mathbb{L} u(\cdot) \varphi_{i}(\cdot)\right\rangle_{W_{2}^{1}[a, b]} \\
& \quad=(\mathbb{L} u)\left(x_{i}\right)=0 .
\end{aligned}
$$

Assume that $\left\{x_{i}\right\}_{i=1}^{\infty}$ is dense on $[a, b]$ and so $(\mathbb{L} u)(x)=0$. It follows that $u \equiv 0$ from the existence of $\mathbb{L}^{-1}$. Now, the theorem is proved.

Theorem 9. If $\left\{x_{i}\right\}_{i=1}^{\infty}$ is dense on $[a, b]$ and the solution of (12) is unique, then the solution of (12) is

$$
u(x)=\sum_{i=1}^{\infty} \sum_{k=1}^{i} \beta_{i k} u\left(x_{k}\right) \bar{\psi}_{i}(x) .
$$

Proof. Using (13), we have

$$
\begin{aligned}
u(x) & =\sum_{i=1}^{\infty}\left\langle u(x), \bar{\psi}_{i}(x)\right\rangle_{W_{2}^{1}[a, b]} \bar{\psi}_{i}(x) \\
& =\sum_{i=1}^{\infty}\left\langle u(x), \sum_{k=1}^{i} \beta_{i k} \psi_{k}(x)\right\rangle_{W_{2}^{1}[a, b]} \bar{\psi}_{i}(x) \\
& =\sum_{i=1}^{\infty} \sum_{k=1}^{i} \beta_{i k}\left\langle u(x), \psi_{k}(x)\right\rangle_{W_{2}^{1}[a, b]} \bar{\psi}_{i}(x) \\
& =\sum_{i=1}^{\infty} \sum_{k=1}^{i} \beta_{i k}\left\langle u(x), \mathbb{L}^{*} \varphi_{k}(x)\right\rangle_{W_{2}^{1}[a, b]} \bar{\psi}_{i}(x) \\
& =\sum_{i=1}^{\infty} \sum_{k=1}^{i} \beta_{i k}\left\langle\mathbb{L} u(x), \varphi_{k}(x)\right\rangle_{W_{2}^{1}[a, b]} \bar{\psi}_{i}(x) \\
& =\sum_{i=1}^{\infty} \sum_{k=1}^{i} \beta_{i k} \llbracket u\left(x_{k}\right) \bar{\psi}_{i}(x) \\
& =\sum_{i=1}^{\infty} \sum_{k=1}^{i} \beta_{i k} u\left(x_{k}\right) \bar{\psi}_{i}(x) .
\end{aligned}
$$

On the other hand, $u(x) \in W_{2}^{1}[a, b]$ and $u(x)=\sum_{i=0}^{\infty} \widehat{a}_{i} \bar{\psi}_{i}(x)$, $\widehat{a}_{i}=\left\langle u(x), \bar{\psi}_{i}(x)\right\rangle$, are the Fourier series expansion about 
normal orthogonal system $\left\{\bar{\psi}_{i}(x)\right\}_{i=1}^{\infty}$ and $W_{2}^{1}[a, b]$ is the Hilbert Space. Thus the series $\sum_{i=0}^{\infty} \hat{a}_{i} \bar{\psi}_{i}(x)$ is convergent in the sense of $\|\cdot\|_{W_{2}^{1}}$ and the proof would be complete.

Now the approximate solution $u_{n}(x)$ can be obtained by the $n$-term intercept of the exact solution $u(x)$ and

$$
u_{n}(x)=\sum_{i=1}^{n} \sum_{k=1}^{i} \beta_{i k} u\left(x_{k}\right) \bar{\psi}_{i}(x) .
$$

Theorem 10. If $u(x) \in W_{2}^{1}[a, b]$ then $M>0$ exists such that $|u(x)| \leq M\|u(x)\|_{W_{2}^{1}[a, b]}$.

Proof. We have $u(x)=\left\langle u(t), R_{x}(t)\right\rangle_{W_{2}^{1}[a, b]}$, for any $x, t$ $\in[a, b]$. We know $\left\|R_{x}(t)\right\|_{W_{2}^{1}[a, b]} \leq M$. Thus $|u(x)|=\mid\langle u(t)$, $\left.R_{x}(t)\right\rangle_{W_{2}^{1}[a, b]} \mid \leq\left\|R_{x}(t)\right\|_{W_{2}^{1}[a, b]}\|u(t)\|_{W_{2}^{1}[a, b]} \leq M\|u(t)\|_{W_{2}^{1}[a, b]}$.

Theorem 11. The approximate solution $u_{n}(x)$ is uniformly convergent.

Proof. Assuming $x \in[a, b]$, by Theorems 9 and 10, it can be proved that

$$
\begin{aligned}
& \lim _{n \rightarrow \infty}\left|u_{n}(x)-u(x)\right| \\
& \quad=\lim _{n \rightarrow \infty}\left|\left\langle u_{n}(x)-u(x), R_{x}(x)\right\rangle_{W_{1}^{2}[a, b]}\right| \\
& \quad \leq M \lim _{n \rightarrow \infty}\left\|u_{n}(x)-u(x)\right\|_{W_{1}^{2}[a, b]}=0 .
\end{aligned}
$$

In the sequel, a new iterative method to achieve the solution of (12) is presented. If

$$
A_{i}=\sum_{k=1}^{i} \beta_{i k} u\left(x_{k}\right)
$$

then (16) can be written as

$$
u(x)=\sum_{i=1}^{\infty} A_{i} \bar{\psi}_{i}(x)
$$

Now suppose, for some $x_{j}, u\left(x_{j}\right)$ is known. There is no problem if we assume $j=1$. We put $u_{0}\left(x_{1}\right)=u\left(x_{1}\right)$ and define the $n$-term approximation to $u(x)$ by

$$
u_{n}(x)=\sum_{i=1}^{n} B_{i} \bar{\psi}_{i}(x)
$$

where

$$
B_{i}=\sum_{k=1}^{i} \beta_{i k} u_{k-1}\left(x_{k}\right) \text {. }
$$

In the following, it would be proven that the approximate solution $u_{n}(x)$ in the iterative $(22)$ is convergent to the exact solution of (12) uniformly.
Theorem 12. Suppose that $\left\|u_{n}\right\|_{W_{1}^{2}[a, b]}$ is bounded in (22). If $\left\{x_{i}\right\}_{i=1}^{\infty}$ is dense on $[a, b]$ then $n$-term approximate solution $u_{n}(x)$ in the iterative (22) converges to the exact solution $u(x)$ of (12) and $u(x)=\lim _{n \rightarrow \infty} \sum_{i=1}^{n} B_{i}^{N} \psi_{i}(x)$, whereas $B_{i}$ is given by (23).

Proof. First of all, the convergence of $u_{n}(x)$ from (22) would be proven. We infer

$$
u_{n+1}(x)=u_{n}(x)+B_{n+1} \bar{\psi}_{n+1}(x) .
$$

Subsequence $\left\{\bar{\psi}_{i}(x)\right\}_{i=1}^{\infty}$ is orthogonal, and it yields that

$$
\left\|u_{n+1}\right\|_{W_{1}^{2}[a, b]}^{2}=\left\|u_{n}\right\|_{W_{1}^{2}[a, b]}^{2}+B_{n+1}^{2}=\sum_{i=1}^{n} B_{i}^{2} .
$$

It is obvious that the sequence $\left\|u_{n}\right\|_{W_{1}^{2}[a, b]}$ is monotonically increasing. Because $\left\|u_{n}\right\|_{W_{1}^{2}[a, b]}$ is bounded and $\left\|u_{n}\right\|_{W_{1}^{2}[a, b]}$ is convergent, then $\sum_{i=1}^{\infty} B_{i}^{2}$ is bounded and this implies that $\left\{B_{i}\right\}_{i=1}^{\infty} \in l^{2}$.

If $m>n$ then

$$
\begin{aligned}
\left\|u_{m}-u_{n}\right\|_{W_{1}^{2}[a, b]}^{2} & =\left\|\sum_{i=m}^{n+1}\left(u_{i}-u_{i-1}\right)\right\|_{W_{1}^{2}[a, b]}^{2} \\
& =\sum_{i=m}^{n+1}\left\|u_{i}-u_{i-1}\right\|_{W_{1}^{2}[a, b]}^{2} .
\end{aligned}
$$

So $\left\|u_{i}-u_{i-1}\right\|_{W_{1}^{2}[a, b]}^{2}=B_{i}^{2}$. Consequently $\left\|u_{m}-u_{n}\right\|_{W_{1}^{2}[a, b]}^{2}=$ $\sum_{i=m}^{n+1} B_{i}^{2} \rightarrow 0$ as $n \rightarrow \infty$. To prove the completeness of $W_{2}^{1}[a, b]$ it requires $\widehat{u}$, where $\widehat{u} \in W_{2}^{1}[a, b]$ that $u_{n} \rightarrow \widehat{u}$ as $n \rightarrow \infty$. Now we can prove $\widehat{u}$ is the solution of (12).

If we take limit from (22), we will have $\widehat{u}(x)=$ $\sum_{i=1}^{n} B_{i} \bar{\psi}_{i}(x)$, so $\mathbb{u} \widehat{u}(x)=\sum_{i=1}^{\infty} B_{i} \llbracket \bar{\psi}_{i}(x)$. Let $x_{l} \in\left\{x_{i}\right\}_{i=1}^{\infty}$, and then

$$
\begin{aligned}
\mathbb{L} \widehat{u}\left(x_{l}\right) & =\sum_{i=1}^{\infty} B_{i} \mathbb{L} \bar{\psi}_{i}\left(x_{l}\right) \\
& =\sum_{i=1}^{\infty} B_{i}\left\langle\llbracket \bar{\psi}_{i}(x), \varphi_{l}(x)\right\rangle_{W_{2}^{1}[a, b]} \\
& =\sum_{i=1}^{\infty} B_{i}\left\langle\bar{\psi}_{i}(x), \mathbb{L}^{*} \varphi_{l}(x)\right\rangle_{W_{2}^{1}[a, b]} \\
& =\sum_{i=1}^{\infty} B_{i}\left\langle\bar{\psi}_{i}(x), \bar{\psi}_{l}(x)\right\rangle_{W_{2}^{1}[a, b]} \\
& =\sum_{i=1}^{\infty} B_{i}\left\langle\bar{\psi}_{i}(x), \sum_{l=1}^{i} B_{i l} \psi_{l}(x)\right\rangle_{W_{2}^{1}[a, b]} .
\end{aligned}
$$

From (23) and (29), it is concluded that $\mathbb{\imath} \widehat{u}\left(x_{l}\right)=\widehat{u}\left(x_{l}\right)$.

$\left\{x_{i}\right\}_{i=1}^{\infty}$ is dense on $[a, b]$. For each $x \in[a, b],\left\{x_{n_{i}}\right\}_{i=1}^{\infty}$ subsequence exists that $x_{n_{i}} \rightarrow x$ as $i \rightarrow \infty$. Hence, when $i \rightarrow \infty$, we have $\mathbb{L} u(x)=u(x)$ which indicates that $\widehat{u}$ is the solution of (12). 
The mentioned scheme above is an efficient method of solving nonlinear equations [31-33]. However, in implementing this algorithm on a computer, $\left\{\bar{\psi}_{i}(x)\right\}_{i=1}^{\infty}$ is not quite orthogonal, due to rounding errors. In other words, GramSchmidt process is numerically unstable and the computational cost of the algorithm is high. Therefore, the following process is suggested similar for linear problems in [20,34]. This is the subject of the next theorem, where the following notations are used:

$$
\begin{aligned}
& \boldsymbol{\beta}_{n}=\left[\begin{array}{cccc}
\beta_{11} & 0 & \cdots & 0 \\
\beta_{21} & \beta_{22} & \ddots & 0 \\
\vdots & \vdots & \ddots & \vdots \\
\beta_{n 1} & \beta_{n 2} & \cdots & \beta_{n n}
\end{array}\right], \\
& \psi_{n}=\left[\begin{array}{cccc}
\psi_{11} & \psi_{12} & \cdots & \psi_{1 n} \\
\psi_{21} & \psi_{22} & \ddots & \psi_{2 n} \\
\vdots & \vdots & \ddots & \vdots \\
\psi_{n 1} & \psi_{n 2} & \cdots & \psi_{n n}
\end{array}\right] \text {, } \\
& \boldsymbol{\Lambda}=\boldsymbol{\beta}^{\mathrm{T}} \mathbf{B} . \\
& \left\langle\mathbb{L} u_{n}(x), \bar{\psi}_{i}\right\rangle=\left\langle u_{n}(x), \bar{\psi}_{i}\right\rangle \Longrightarrow \\
& \sum_{j=1}^{n} B_{j}\left\langle\mathbb{L} \bar{\psi}_{j}, \bar{\psi}_{i}\right\rangle=\left\langle u_{n}(x), \bar{\psi}_{i}\right\rangle .
\end{aligned}
$$$$
\mathbf{u}=\left[\begin{array}{c}
u_{0}\left(x_{1}\right) \\
u_{1}\left(x_{2}\right) \\
\vdots \\
u_{n-1}\left(x_{n}\right)
\end{array}\right],
$$$$
\mathbf{B}=\left[\begin{array}{c}
B_{1} \\
B_{2} \\
\vdots \\
B_{n}
\end{array}\right],
$$$$
\boldsymbol{\Lambda}=\left[\begin{array}{c}
\Lambda_{1} \\
\Lambda_{2} \\
\vdots \\
\Lambda_{n}
\end{array}\right] \text {, }
$$$$
n=1,2, \ldots
$$

Theorem 13 (let $\gamma_{i j}=\left[\psi^{-1}\right]_{i j}$ ). The approximate solution obtained from (22) is found as follows:

$$
u_{n}(x)=\sum_{i=1}^{n} \Lambda_{i} \psi_{i}(x)
$$

where

$$
\Lambda_{i}=\sum_{k=1}^{i} \gamma_{i k} u_{k-1}\left(x_{k}\right)
$$

Proof. Suppose that $u_{n}(x)=\sum_{i=1}^{n} \Lambda_{i} \psi_{i}(x)=\sum_{i=1}^{n} B_{i} \bar{\psi}_{i}(x)$. Since $\bar{\psi}_{i}(x)=\sum_{k=1}^{i} \beta_{i k} \psi_{k}(x)$,

$$
\begin{aligned}
\sum_{i=1}^{n} \Lambda_{i} \psi_{i}(x) & =\sum_{i=1}^{n} B_{i} \bar{\psi}_{i}(x)=\sum_{i=1}^{n} B_{i} \sum_{k=1}^{i} \beta_{i k} \psi_{k}(x) \\
& =\sum_{k=1}^{n} \sum_{i=k}^{n} B_{i} \beta_{i k} \psi_{k}(x) .
\end{aligned}
$$

$\left\{\psi_{i}(x)\right\}_{i=1}^{\infty}$ and $\Lambda_{k}=\sum_{i=k}^{n} B_{i} \beta_{i k}(k=1,2, \ldots, n)$ are linear

Both sides of (33) provide

$$
\begin{aligned}
\sum_{j=1}^{n} B_{j}\left\langle\mathbb{L} \bar{\psi}_{i}, \bar{\psi}_{i}\right\rangle & =\sum_{j=1}^{n} B_{j} \sum_{k=1}^{i} \beta_{i k} \sum_{l=1}^{j} \beta_{j l}\left\langle\mathbb{L} \psi_{l}, \psi_{k}\right\rangle \\
& =\sum_{j=1}^{n} B_{j} \sum_{k=1}^{i} \sum_{l=1}^{j} \beta_{i k}\left\langle\mathbb{L} \psi_{l}, \psi_{k}\right\rangle \beta_{l j}^{T} \\
& =\sum_{j=1}^{n} B_{j}\left(\beta \psi \beta^{\mathrm{T}}\right)_{i j}, \\
\left\langle u_{n}(x), \bar{\psi}_{i}\right\rangle & =B_{i}=\sum_{k=1}^{i} \beta_{i k} u_{k-1}\left(x_{k}\right),
\end{aligned}
$$

(using Theorem 12)

From (32) and (35) the following equation can be reached:

$$
\beta \psi \beta^{\mathrm{T}} \mathbf{B}=\beta \mathbf{u} .
$$

Equation (32) implies $\boldsymbol{\beta} \psi \boldsymbol{\Lambda}=\boldsymbol{\beta} \mathbf{u}_{n}$. So

$$
\psi \Lambda=\mathbf{u}_{n},
$$

which proves the theorem.

Algorithm 14. The following steps exist for approximating the solution without applying Gram-Schmidt orthogonal process:

Step 1. Fix $a \leq x$ and $t \leq b$.

If $t \leq x$, set $R_{x}(t)=1-a+t$.

Else set $R_{x}(t)=1-a+x$.

Step 2. For $i=1,2, \ldots, m$ set $x_{i}=(i-1) /(m-1)$. 
TABLE 1: The absolute errors comparison between the proposed approach and method [30].

\begin{tabular}{cccccc}
\hline$x$ & Exact solution & Approximate solution & Method in [30] & Absolute error & Absolute error in method [30] \\
\hline 0.1 & 1.105170918 & 1.105154993 & 1.114627560 & 0.000015925 & 0.009456642 \\
0.2 & 1.221402757 & 1.221389903 & 1.226758840 & 0.000012855 & 0.005356083 \\
0.3 & 1.349858806 & 1.349845490 & 1.345458179 & 0.000013317 & 0.004400627 \\
0.4 & 1.491824696 & 1.491803913 & 1.480202470 & 0.000020784 & 0.011622226 \\
0.5 & 1.648721268 & 1.648680858 & 1.671769819 & 0.000040412 & 0.023048551 \\
0.6 & 1.822118797 & 1.822039541 & 1.838854903 & 0.000079259 & 0.016736106 \\
0.7 & 2.013752703 & 2.013608464 & 2.022118086 & 0.000144243 & 0.008365383 \\
0.8 & 2.225540923 & 2.225304288 & 2.223139077 & 0.000236640 & 0.002401846 \\
0.9 & 2.459603104 & 2.459253211 & 2.443684898 & 0.000349899 & 0.015918206 \\
\hline
\end{tabular}

TABLE 2: The absolute errors comparison between the proposed approach and method [26].

\begin{tabular}{cccccc}
\hline$x$ & Exact solution & Approximate solution & Method in [26] & Absolute error & Absolute error in method [26] \\
\hline 0.1 & 0.099833 & 0.098524 & 0.101388 & 0.001308 & 0.00155 \\
0.2 & 0.198669 & 0.199766 & 0.194073 & 0.001097 & 0.004596 \\
0.3 & 0.295520 & 0.294857 & 0.299995 & 0.000662 & 0.004475 \\
0.4 & 0.389418 & 0.389658 & 0.387978 & 0.000240 & 0.001440 \\
0.5 & 0.479426 & 0.479585 & 0.486266 & 0.000160 & 0.006840 \\
0.6 & 0.564642 & 0.563993 & 0.565930 & 0.000648 & 0.001288 \\
0.7 & 0.644218 & 0.645477 & 0.640624 & 0.001260 & 0.003594 \\
0.8 & 0.717356 & 0.715373 & 0.720611 & 0.001982 & 0.003255 \\
0.9 & 0.783327 & 0.785653 & 0.782351 & 0.002326 & 0.000976 \\
\hline
\end{tabular}

Set $\psi_{i}(x)=\left.L_{t} R_{x}(t)\right|_{t=x_{i}}$.

Step 3. Set $u_{0}\left(x_{1}\right)=u\left(x_{1}\right)$.

Step 4. For $i=1,2, \ldots, m$ set $\gamma_{i j}=\left[\psi^{-1}\right]_{i j}$.

Step $5 . n=1$.

Step 6. Set $\Lambda_{n}=\sum_{k=1}^{n} \gamma_{n k} u_{k-1}\left(x_{k}\right)$.

Step 7. Set $u_{n}(x)=\sum_{i=1}^{n} \Lambda_{i} \psi_{i}(x)$.

Step 8. If $n<m$ then set $n=n+1$ and go to step 6 .

Else stop.

Algorithm 15. The following steps exist for approximating the solution by applying Gram-Schmidt orthogonal process:

Step 1. Fix $a \leq x$ and $t \leq b$.

If $t \leq x$, set $R_{x}(t)=1-a+t$.

Else set $R_{x}(t)=1-a+x$.

Step 2. For $i=1,2, \ldots, m$ set $x_{i}=(i-1) /(m-1)$.

Set $\psi_{i}(x)=\left.L_{t} R_{x}(t)\right|_{t=x_{i}}$.

Step 3. For $i=1,2, \ldots, m$ and $j=$ $1,2, \ldots, m$, if $i \neq j$ then set $\beta_{i j}=$ $\left(-1 / \sqrt{\left\|\psi_{i}\right\|^{2}-\sum_{k=1}^{i-1}\left\langle\psi_{i}(t), \bar{\psi}_{k}(t)\right\rangle^{2}}\right) \sum_{k=1}^{i-1}\left\langle\psi_{i}(t) \quad\right.$ and $\left.\bar{\psi}_{k}(t)\right\rangle \beta_{k j}$.

Else $\beta_{i j}=1 / \sqrt{\left\|\psi_{i}\right\|^{2}-\sum_{k=1}^{i-1}\left\langle\psi_{i}(t), \bar{\psi}_{k}(t)\right\rangle^{2}}$.

Else $\beta_{11}=1 /\left\|\psi_{1}\right\|$.
Step 4. For $i=1,2, \ldots, m$ set $\bar{\psi}_{i}(x)=\sum_{k=1}^{i} \beta_{i k} \psi_{k}(x)$.

Step 5. Set $u_{0}\left(x_{1}\right)=u\left(x_{1}\right)$.

Step 6. Set $n=1$.

Step 7. Set $B_{n}=\sum_{k=1}^{n} \beta_{n k} u_{k-1}\left(x_{k}\right)$.

Step 8. Set $u_{n}(x)=\sum_{i=1}^{n} B_{i} \bar{\psi}_{i}(x)$.

Step 9. If $n<m$ then set $n=n+1$ and go to step 7 .

Else stop.

\section{Numerical Experiments}

In this part, four numerical examples are solved for potency and utility of the present method. All computations are performed by MAPLE package. Results which are taken by this method show a proper agreement with the exact solution. A comprehensive applicability of this method is given the stability and consistence of the presented method. The reliability of the method and increasing of the accuracy cause this method to be more applicable.

Example 1. For first applicable instance, we offer nonlinear Fredholm integral equations $[26,30]$ :

$$
u(x)-\int_{0}^{1} x y u^{3}(y) d y=e^{x}-\frac{\left(1+2 e^{3}\right) x}{9},
$$



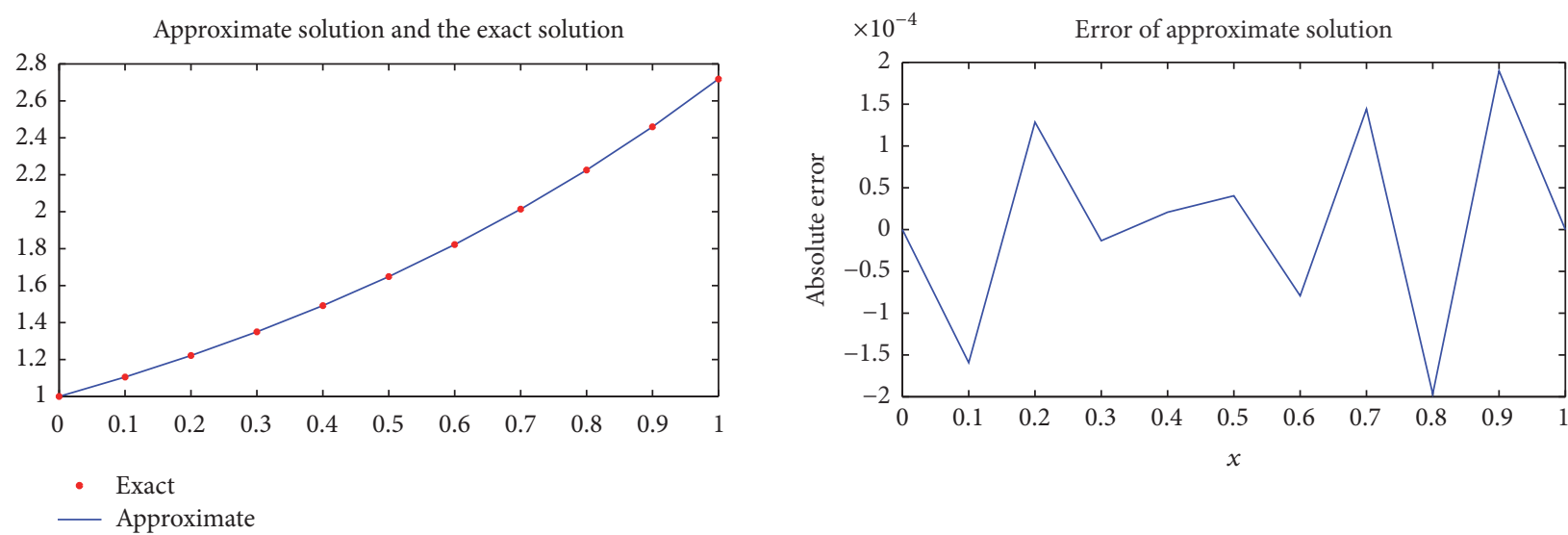

Figure 1: The absolute errors comparison between the proposed approach and method [30].
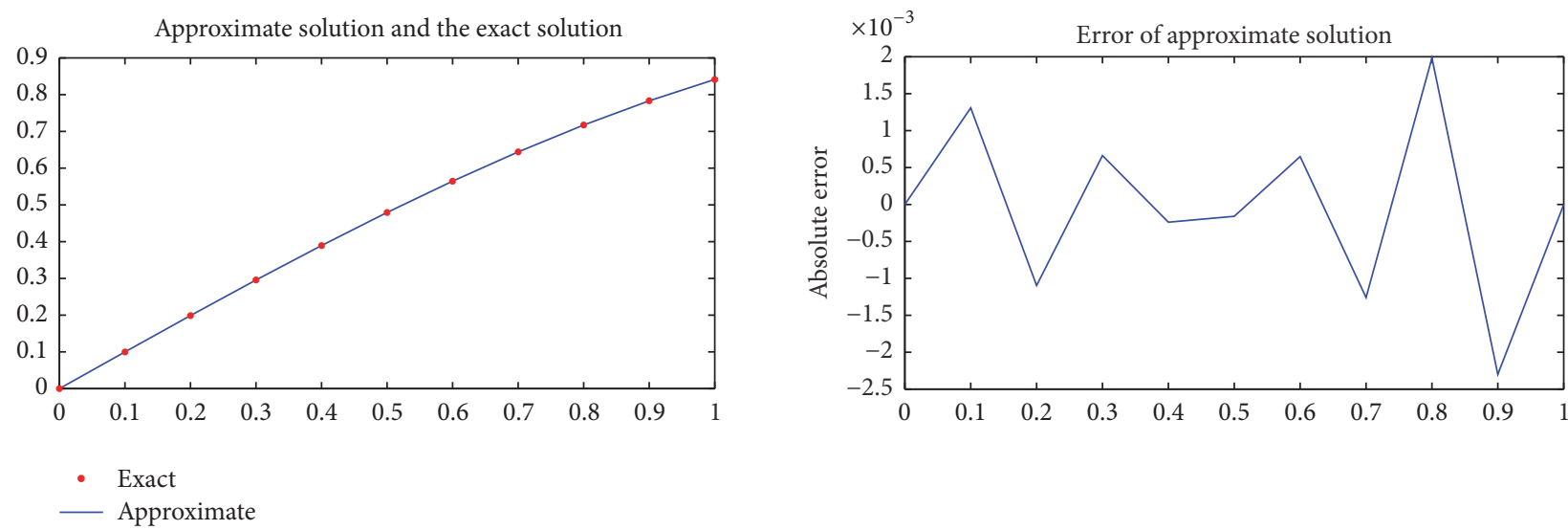

FIGURE 2: The absolute errors comparison between the proposed approach and method [26].

The exact solution of this equation is $u(x)=e^{x}$. According to (38) we can assume an initial approximation $u_{0}(0)=u(0)=$ 1. The numerical results are given in Table 1 by taking $x_{i}=$ $(i-1) /(n-1), i=1,2, \ldots, n$ and $n=128$. In Table 1 a comparison between the absolute errors of our method and the Haar wavelet method [30] is given. Figure 1 shows the approximate solution and its errors.

Example 2. For second applicable example, an electromagnetic problem is solved via the presented method. It is simulated to nonlinear Volterra integral equations model $[26,30]$ :

$$
\begin{array}{r}
u(x)-\frac{1}{2} \int_{0}^{x} u^{2}(y) d y=\sin (x)+\frac{1}{8} \sin (2 x)-\frac{1}{4} x, \\
0 \leq x \leq 1 .
\end{array}
$$

The exact solution of this equation is $u(x)=\sin (x)$. According to (39) we can assume an initial approximation $u_{0}(0)=u(0)=0$. Numerical results are given in Table 2 by taking $x_{i}=(i-1) /(n-1), i=1,2, \ldots, n$ and $n=128$. In Table 2 a comparison between the absolute errors of the proposed method and the BPFs method [26] is given. Figure 2 shows
TABLE 3: The execution time (seconds) comparison between Algorithms 14 and 15 .

\begin{tabular}{lcc}
\hline$n$ & Algorithm $14(\mathrm{Sec})$ & Algorithm $15(\mathrm{Sec})$ \\
\hline 4 & 3.47 & 2.87 \\
8 & 9.52 & 4.82 \\
16 & 59.75 & 31.77 \\
\hline
\end{tabular}

the approximate solution and its errors. In Table 3, a comparison execution time between Algorithms 14 and 15 is given.

Example 3. An electromagnetic problem is solved via our method for another applicable example. It is simulated to nonlinear Fredholm integral equations model [26, 30]:

$$
u(x)-\frac{1}{2} \int_{0}^{1} y u^{2}(y) d y=x^{2}-\frac{1}{12}, \quad 0 \leq x \leq 1 .
$$

The exact solution of this equation is $u(x)=x^{2}$. According to $(40)$, the initial approximation $u_{0}(0)=u(0)=0$ is chosen. Numerical results are given in Table 4 by taking $x_{i}=(i-$ 1) $/(n-1), i=1,2, \ldots, n$ and $n=128$. In Table 4 a comparison 

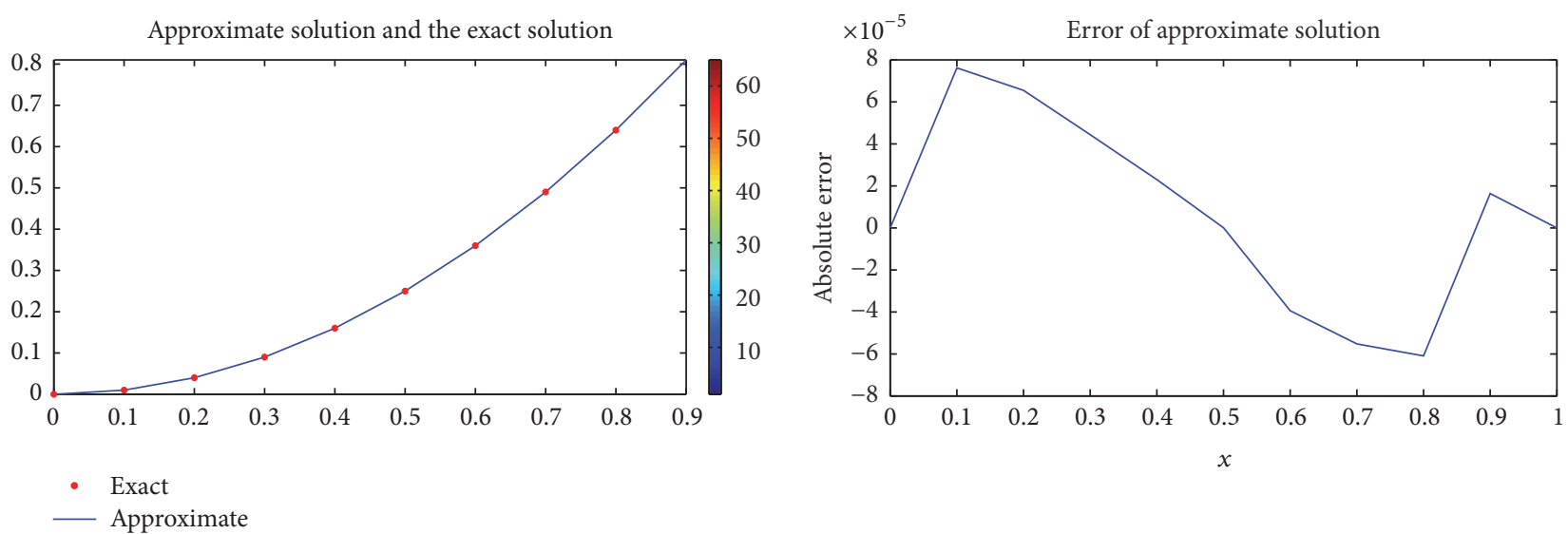

FIGURE 3: The absolute errors comparison between the proposed approach and method [26].

TABLE 4: The absolute errors comparison between the proposed approach and method [26].

\begin{tabular}{|c|c|c|c|c|c|}
\hline$x$ & Exact solution & Approximate solution & Method in [26] & Absolute error & Absolute error in method [26] \\
\hline 0.1 & 0.010000 & 0.010762 & 0.010308 & 0.000762 & 0.000308 \\
\hline 0.2 & 0.040000 & 0.040655 & 0.038140 & 0.000655 & 0.001860 \\
\hline 0.3 & 0.090000 & 0.090444 & 0.092828 & 0.000444 & 0.002828 \\
\hline 0.4 & 0.160000 & 0.16023 & 0.158746 & 0.000230 & 0.001254 \\
\hline 0.5 & 0.250000 & 0.250001 & 0.257867 & 0.000001 & 0.007867 \\
\hline 0.6 & 0.360000 & 0.359606 & 0.361871 & 0.000394 & 0.001871 \\
\hline 0.7 & 0.490000 & 0.489448 & 0.483453 & 0.000552 & 0.006547 \\
\hline 0.8 & 0.640000 & 0.639391 & 0.647515 & 0.000609 & 0.007515 \\
\hline 0.9 & 0.810000 & 0.811638 & 0.807183 & 0.001638 & 0.002817 \\
\hline
\end{tabular}

TABLE 5: The execution time (seconds) comparison between Algorithms 14 and 15.

\begin{tabular}{lcc}
\hline$n$ & Algorithm $14(\mathrm{Sec})$ & Algorithm $15(\mathrm{Sec})$ \\
\hline 4 & 3.1 & 2.59 \\
8 & 8.46 & 5.09 \\
16 & 34.2 & 28.89 \\
\hline
\end{tabular}

between the absolute errors of the proposed method and the BPFs method [26] is given. Figure 3 shows the approximate solution and its error. In Table 5 a comparison execution time between Algorithms 14 and 15 is given.

Example 4. A nonlinear Fredholm integral problem $[26,30]$ is solved via our method for this applicable example:

$$
u(x)+\int_{0}^{1} e^{x-2 y} u^{3}(y) d y=e^{x+1}, \quad 0 \leq x<1
$$

The exact solution of this equation is $u(x)=e^{x}$. According to (41) we consider an initial approximation as $u_{0}(0)=u(0)=1$. Numerical results are given in Table 6 by taking $x_{i}=(i-$ $1) /(n-1), i=1,2, \ldots, n$ and $n=128$. In Table 6 a comparison between the absolute errors of the proposed method and the Haar wavelet method [30] is given. Figure 4 shows the approximate solution and its error.

\section{Conclusion}

According to this essay, supplementary of iterative Reproducing Kernel Hilbert Space Method was introduced and applied to acquire the approximate solution of some nonlinear integral equation. In this method, unlike other similar methods, orthogonal process is not used. However the time is increasing, and accuracy is also increasing. The main point which is mentioned in this paper is that Algorithm 14 has higher execution time in comparison with Algorithm 15, but the approximate solution in Algorithm 14 is more accurate than Algorithm 15. Current uniform convergence method is stated and proved. The obtained numerical results confirm that it is a good candidate for solving the nonlinear integral equation.

\section{Conflicts of Interest}

The authors declare no conflicts of interest. 
TABLE 6: The absolute errors comparison between the proposed approach and method [30].

\begin{tabular}{cccccc}
\hline$x$ & Exact solution & Approximate solution & Method in [30] & Absolute error & Absolute error in method [30] \\
\hline 0.1 & 1.105170918 & 1.105495796 & 1.111099473 & 0.000324878 & 0.005928555 \\
0.2 & 1.221402757 & 1.220931139 & 1.220296615 & 0.000471618 & 0.001106142 \\
0.3 & 1.349858806 & 1.349502887 & 1.339817084 & 0.000355919 & 0.010041722 \\
0.4 & 1.491824696 & 1.491740269 & 1.471965091 & 0.000084427 & 0.019859605 \\
0.5 & 1.648721268 & 1.648649846 & 1.457127691 & 0.000071422 & 0.191593577 \\
0.6 & 1.822118797 & 1.822243106 & 1.831888927 & 0.000124309 & 0.009770130 \\
0.7 & 2.013752703 & 2.013650232 & 2.011936556 & 0.000102471 & 0.001816147 \\
0.8 & 2.225540923 & 2.224684503 & 2.209678381 & 0.000856420 & 0.015862542 \\
0.9 & 2.459603104 & 2.458626573 & 2.426854676 & 0.000976531 & 0.032748428 \\
\hline
\end{tabular}
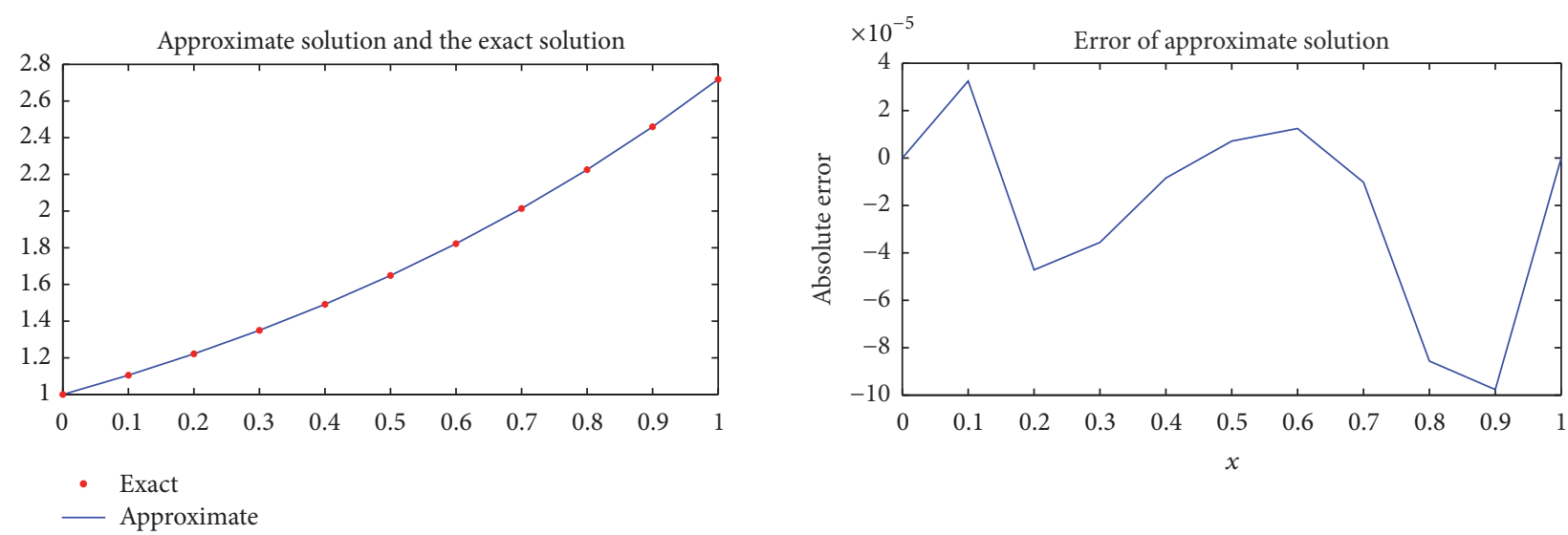

FIGURE 4: The absolute errors comparison between the proposed approach and method [30].

\section{Acknowledgments}

The authors would like to thank Professor Haipeng Peng for instructive comments and recommendations to improve the quality of this work and also the Islamic Azad University, Science and Research Branch, Tehran, for supporting this project.

\section{References}

[1] K. Meetz and W. Engi, Electromagnetische Felder, Springer, Berlin, Germany, 1979.

[2] W. Thirring, Lehrbuch der Mathematischen Physik, vol. 2, Springer, Vienna, Austria, 1978.

[3] K. F. Warnick, R. H. Selfridge, and D. V. Arnold, "Teaching electromagnetic field theory using differential forms," IEEE Transactions on Education, vol. 40, no. 1, pp. 53-68, 1997.

[4] S. Abbasbandy, "Homotopy analysis method for heat radiation equations," International Communications in Heat and Mass Transfer, vol. 34, no. 3, pp. 380-387, 2007.

[5] H. Vosughi, E. Shivanian, and S. Abbasbandy, "A new analytical technique to solve Volterra's integral equations," Mathematical Methods in the Applied Sciences, vol. 34, no. 10, pp. 1243-1253, 2011.

[6] J.-P. Kauthen, "A survey of singularly perturbed Volterra equations," Applied Numerical Mathematics. An IMACS Journal, vol. 24, no. 2-3, pp. 95-114, 1997.
[7] A. A. Kilbas and M. Saigo, "On solution of nonlinear AbelVolterra integral equation," Journal of Mathematical Analysis and Applications, vol. 229, no. 1, pp. 41-60, 1999.

[8] C. Minggen and D. Zhongxing, "On the best operator of interpolation," Mathematica Numerica Sinica, vol. 8, no. 2, pp. 209-216, 1986.

[9] C. Minggen and L. Yingzhen, Nonlinear Numerical Analysis in the Reproducing Kernel Space, Nova Science, New York, NY, USA, 2009.

[10] S. Abbasbandy, B. Azarnavid, and M. S. Alhuthali, "A shooting reproducing kernel Hilbert space method for multiple solutions of nonlinear boundary value problems," Journal of Computational and Applied Mathematics, vol. 279, pp. 293-305, 2015.

[11] N. Shawagfeh, O. Abu Arqub, and S. Momani, "Analytical solution of nonlinear second-order periodic boundary value problem using reproducing kernel method," Journal of Computational Analysis and Applications, vol. 16, no. 4, pp. 750-762, 2014.

[12] F. Geng and M. Cui, "Solving a nonlinear system of second order boundary value problems," Journal of Mathematical Analysis and Applications, vol. 327, no. 2, pp. 1167-1181, 2007.

[13] B. Azarnavid, F. Parvaneh, and S. Abbasbandy, "Picard-reproducing kernel Hilbert space method for solving generalized singular nonlinear Lane-Emden type equations," Mathematical Modelling and Analysis, vol. 20, no. 6, pp. 754-767, 2015.

[14] O. A. Arqub, M. Al-Smadi, and N. Shawagfeh, "Solving Fredholm integro-differential equations using reproducing kernel Hilbert space method," Applied Mathematics and Computation, vol. 219, no. 17, pp. 8938-8948, 2013. 
[15] L. Yang and M. Cui, "New algorithm for a class of nonlinear integro-differential equations in the reproducing kernel space," Applied Mathematics and Computation, vol. 174, no. 2, pp. 942960, 2006.

[16] O. Abu Arqub, M. Al-Smadi, and S. Momani, "Application of reproducing kernel method for solving nonlinear FredholmVolterra integrodifferential equations," Abstract and Applied Analysis, vol. 2012, Article ID 839836, 16 pages, 2012.

[17] S. Bushnaq, B. Maayah, S. Momani, and A. Alsaedi, "A reproducing kernel Hilbert space method for solving systems of fractional integrodifferential equations," Abstract and Applied Analysis, vol. 2014, Article ID 103016, 6 pages, 2014.

[18] M. Inc, A. Akgül, and F. Geng, "Reproducing kernel Hilbert space method for solving Bratu's problem," Bulletin of the Malaysian Mathematical Sciences Society, vol. 38, no. 1, pp. 271287, 2015.

[19] A.-M. Wazwaz, A First Course in Integral Equations, World Scientific, 1997.

[20] S. Javadi, E. Babolian, and E. Moradi, "New implementation of reproducing kernel Hilbert space method for solving a class of functional integral equations," Communications in Numerical Analysis, vol. 2014, Article ID cna-00205, 7 pages, 2014.

[21] W. Jiang and Z. Chen, "Solving a system of linear Volterra integral equations using the new reproducing kernel method," Applied Mathematics and Computation, vol. 219, no. 20, pp. 10225-10230, 2013.

[22] A.-M. Wazwaz, Linear and Nonlinear Integral Equations, Methods and Applications, Springer, Berlin, Germany, 2011.

[23] I. L. El-Kalla, "Convergence of the Adomian method applied to a class of nonlinear integral equations," Applied Mathematics Letters. An International Journal of Rapid Publication, vol. 21, no. 4, pp. 372-376, 2008.

[24] G. Gumah, K. Moaddy, M. Al-Smadi, and I. Hashim, "Solutions to uncertain Volterra integral equations by fitted reproducing kernel Hilbert space method," Journal of Function Spaces, vol. 2016, Article ID 2920463, 11 pages, 2016.

[25] V. Sizikov and D. Sidorov, "Generalized quadrature for solving singular integral equations of Abel type in application to infrared tomography," Applied Numerical Mathematics. An IMACS Journal, vol. 106, pp. 69-78, 2016.

[26] E. Babolian, Z. Masouri, and S. Hatamzadeh-Varmazyar, "New direct method to solve nonlinear Volterra-Fredholm integral and integro-differential equations using operational matrix with block-pulse functions," in Progress in Electromagnetics Research B, vol. 8, pp. 59-76, EMW Publishing, Cambridge, Mass, USA, 2008.

[27] D. Alpay, Ed., Reproducing kernel spaces and applications, vol. 143 of Operator Theory: Advances and Applications, Birkhäuser Basel, 2003.

[28] S. Saitoh, D. Alpay, J. A. Ball, and T. Ohsawa, Reproducing Kernels and Their Applications, vol. 11, Springer Science \& Business Media, Berlin, Germany, 2013.

[29] S. Saitoh, Integral Transforms, Reproducing Kernels and Their Applications, vol. 369 of Pitman Research Notes in Mathematics Series, Longman, Harlow, UK, 1997.

[30] E. Babolian and A. Shahsavaran, "Numerical solution of nonlinear Fredholm integral equations of the second kind using Haar wavelets," Journal of Computational and Applied Mathematics, vol. 225, no. 1, pp. 87-95, 2009.

[31] R. Ketabchi, R. Mokhtari, and E. Babolian, "Some error estimates for solving Volterra integral equations by using the reproducing kernel method," Journal of Computational and Applied Mathematics, vol. 273, pp. 245-250, 2015.

[32] A. Alvandi, M. Paripour, and Z. Roshani, "Reproducing kernel method for solving a class of Fredholm integro-differential equations," in Proceedings of the 46th Annual Iranian Mathematics Conference (AIMC 46), p. 505, Yazd University, 2015.

[33] I. Komashynska and M. Al-Smadi, "Iterative reproducing kernel method for solving second-order integrodifferential equations of fredholm type," Journal of Applied Mathematics, vol. 2014, Article ID 459509, 11 pages, 2014.

[34] E. Babolian, S. Javadi, and E. Moradi, "Error analysis of reproducing kernel Hilbert space method for solving functional integral equations," Journal of Computational and Applied Mathematics, vol. 300, pp. 300-311, 2016. 


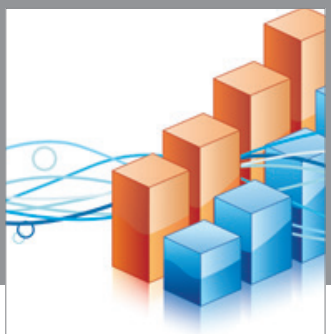

Advances in

Operations Research

vatem alat4

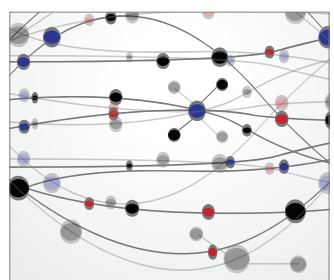

\section{The Scientific} World Journal
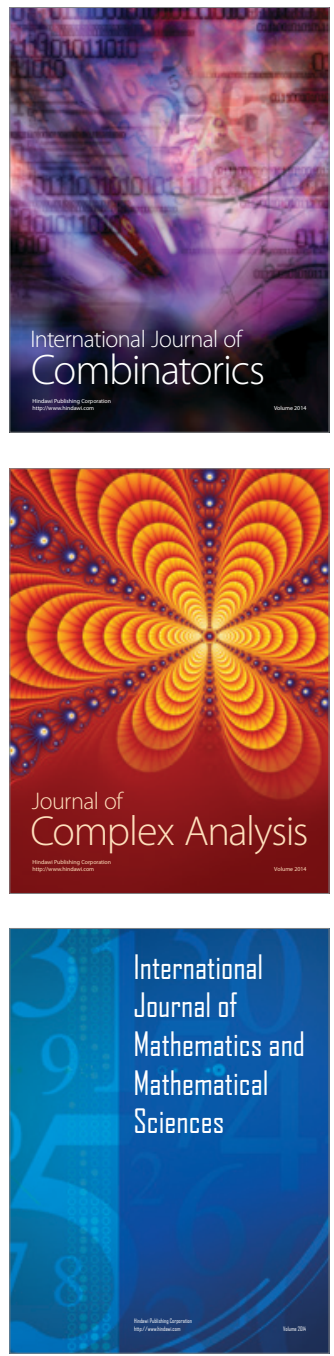
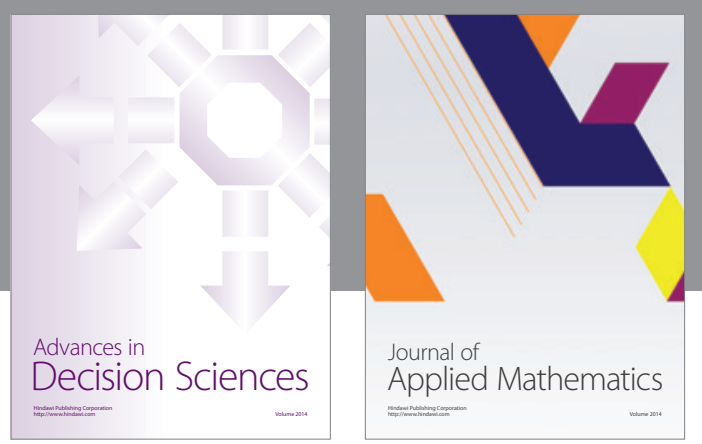

Algebra

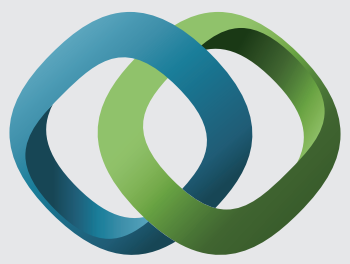

\section{Hindawi}

Submit your manuscripts at

https://www.hindawi.com
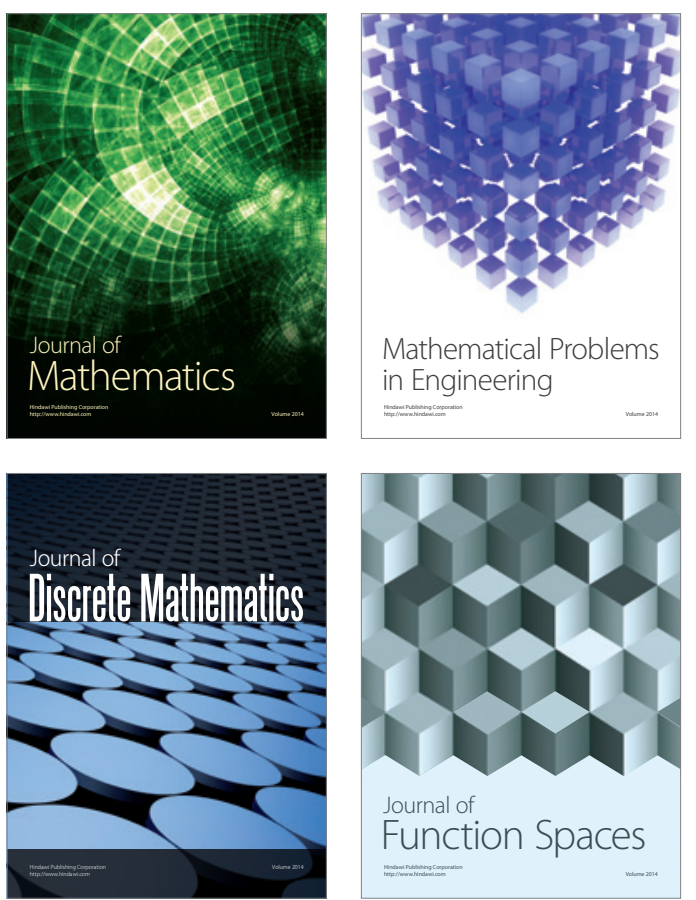

Mathematical Problems in Engineering
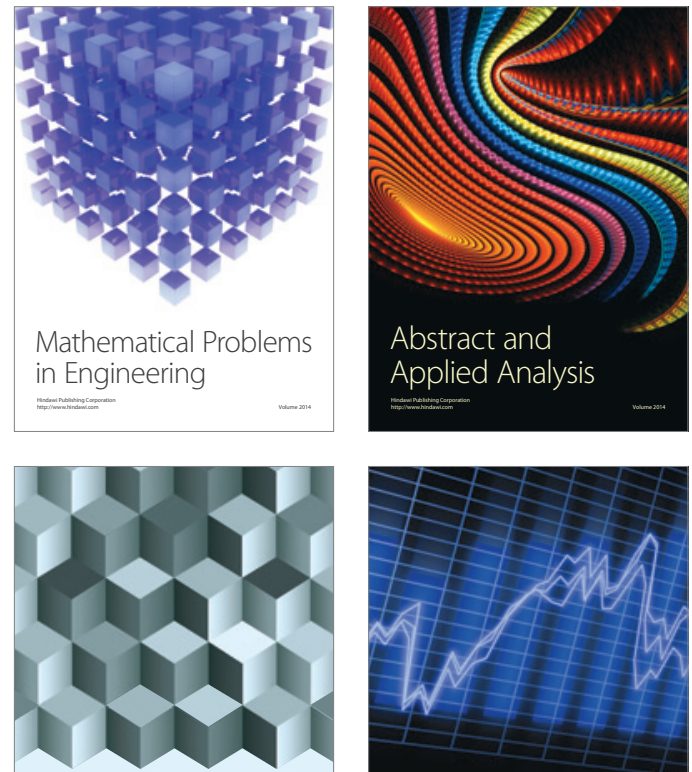

Journal of

Function Spaces

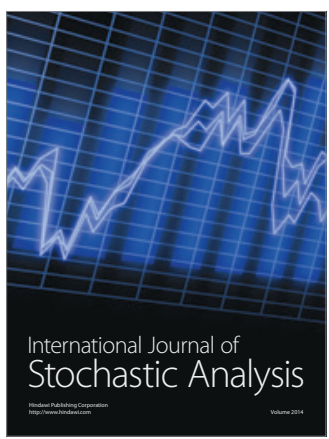

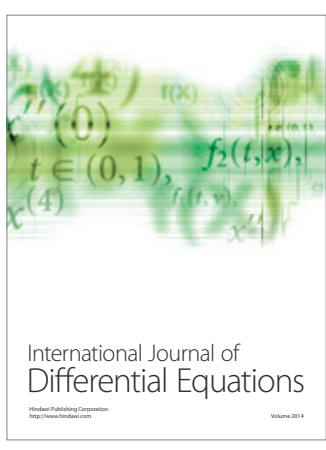
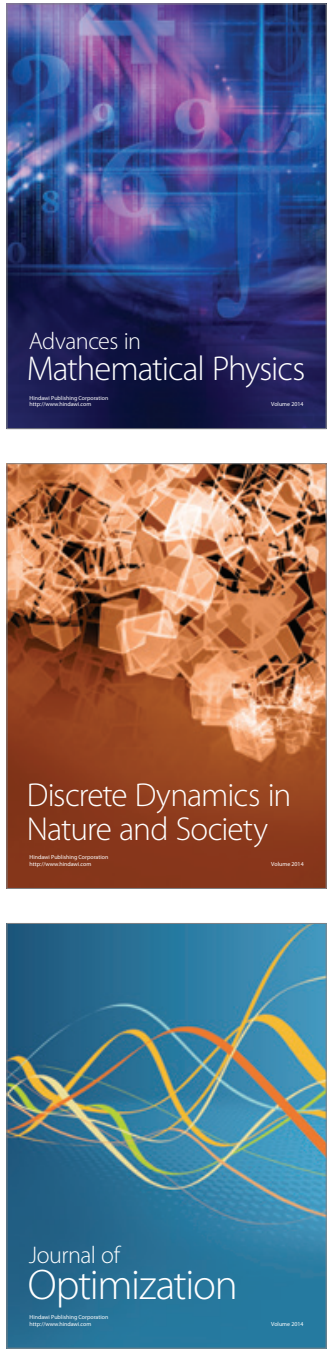